\title{
Motivational interviewing in an oral health promotion programme
}

\author{
Abstracted from \\ Almomani F, Williams K, Catley D, Brown C. \\ Effects of an oral health promotion program in people with mental illness. \\ J Dent Res 2009; 88: 648-652 \\ Address for correspondence: Fidaa Almomani, Jordan University of Science and Technology, \\ Faculty of Applied Medical Sciences, PO Box 3030, Irbid 22110, Jordan. E-mail: falmomani@just.edu.jo
}

\section{Question: Does motivational interviewing immediately before an oral health education session enhance the education effect?}

Design This was a randomised controlled trial (RCT).

Intervention From a group of patients with mental health problems participants in the motivational interviewing (MI) arm received a brief Ml session (15-20 min) conducted by a doctoral psychology student trained in $\mathrm{Ml}$, prior to an oral health education session which was focused on exploring advantages and disadvantages, motivation and confidence, and personal values related to daily toothbrushing and oral health. All participants received two pamphlets summarising the information from the education session, instruction in using a mechanical toothbrush, a reminder system, and weekly telephone calls (for 4 weeks).

Outcome measure Plaque scores, oral health knowledge and selfregulation were assessed at baseline and at 4 and 8 weeks.

Results Sixty participants consented, and 56 (93\%) completed the study. Those who did not complete the study discontinued for personal reasons (eg, moving, hospitalisation). Repeated-measures analysis of variance showed improvement $(P<0.05)$ in plaque levels, autonomous regulation and oral health knowledge over time for both groups. Individuals receiving Ml improved significantly more, however, compared with people receiving oral health education alone.

Conclusions Results suggest that Ml is effective at enhancing shortterm oral health behaviour change for people with severe mental illness and may be useful for the general population.

\section{Commentary}

Mental illness and the various medications used to treat the illness can cause a range of oral complications and side-effects, with dental caries, periodontal diseases and xerostomia encountered most frequently. People with mental illness, however, are often excluded from health promotion activities as they are perceived to be a nuisance. ${ }^{1}$

Traditionally, in healthcare settings, recommendations for behaviour change are delivered through a brief advice-giving in which overt recommendations are provided. ${ }^{2}$ MI challenges traditional intervention-delivery methods by suggesting that patients know what is best for themselves, and that professionals should work with them to determine what behaviour-change strategies will be most effective, while acknowledging freedom of choice. It has been applied to a number of areas of behavioural change, and is best known for applications in the realm of substance abuse. ${ }^{3}$

MI has proved useful for improving health behaviour, including among people who have severe mental illness. This study therefore tested whether MI enhanced the efficacy of an oral health education intervention in individuals with severe mental illness. Patients were recruited from a community programme and a flow diagram was used to depict the passage of them through the RCT. The reporting of the RCT followed closely with the recommendations from the CONSORT (Consolidated Standards of Reporting Trials), statement. ${ }^{4}$

The authors stated that the sample size was determined through power analysis. Variance estimates for the primary outcome measure, plaque scores, were based on their previous study, ${ }^{5}$ with a meaningful difference of $15 \%$ set for effect size, with alpha at 0.05 and power at 0.80 . No details were given, however, about the minimum number of participants required for each intervention group and whether attrition was included in the power calculation. As a result, it would be difficult to draw a conclusion about whether the final number of participants at week 8 was sufficient to test the hypothesis. In addition, it is a good practice to state in the paper whether intention-to-treat statistical analysis has been carried out, even though the number of dropouts was low in this study.

The authors themselves identified two main limitations of this study. Firstly, the followup period was short and so it would be difficult to be certain whether the changes could be maintained after the first 2 months. The other limitation was the additive design, resulting in the MI group receiving more practitioner time. This would hinder the ability to conclude that MI per se (rather than greater attention) led to the observed treatment effects. 
MI is a promising intervention to encourage positive health behaviour change in medical and dental settings but published literature remains scarce on MI in relation to oral health. ${ }^{3,6}$ Clearly, more research is needed with a breadth of populations to contribute to the research base of MI in oral healthcare.

\section{Albert Yeung}

Department of Public Health, NHS Lanarkshire, Hamilton, Scotland, UK

\section{Practice points}

- Ml can lead, at least in the short term, to improved knowledge and oral hygiene in people who have severe mental illness.
1. Armstrong E. Mental Health Issues in Primary Care: a Practical Guide. Basingstoke: Macmillan; 1995

2. Rollnick S, Mason P, Butler C. Health Behaviour Change: a Guide for Practitioners. Edinburgh: Churchill Livingstone; 1999.

3. Martins RK, McNeil DW. Review of motivational interviewing in promoting health behaviors. Clin Psychol Rev 2009; 29: 283-293.

4. Altman DG, Schulz KF, Moher D, et al. The revised CONSORT statement for reporting randomized trials: explanation and elaboration. Ann Intern Med 2001; 134: 663-694.

5. Almomani F, Brown C, Williams KB. The effect of an oral health promotion program for people with psychiatric disabilities. Psychiatr Rehabil J 2006; 29: 274-281.

6. Yevlahova D, Satur J. Models for individual oral health promotion and their effectiveness: a systematic review. Aust Dent / 2009; 54: 190-197.

Evidence-Based Dentistry (2010) 11, 14-15. doi:10.1038/sj.ebd.6400703 